BULLETIN (New Series) OF THE

AMERICAN MATHEMATICAL SOCIETY

Volume 48, Number 2, April 2011, Pages 259-274

S 0273-0979(2011)01329-5

Article electronically published on February 7, 2011

\title{
THE EVOLUTION OF GEOMETRIC STRUCTURES ON 3-MANIFOLDS
}

\author{
CURTIS T. MCMULLEN
}

\begin{abstract}
This paper gives an overview of the geometrization conjecture and approaches to its proof.
\end{abstract}

\section{Contents}

1. Introduction 259

2. Surfaces and tilings 260

3. The geometrization conjecture for 3-manifolds 262

4. Knots 265

5. Evolving geometric structures 266

6. Open problems 270

7. Notes and references 271

About the author $\quad 272$

References $\quad 272$

\section{INTRODUCTION}

In 1300, Dante described a universe in which the concentric terraces of hellnesting down to the center of the earth - are mirrored by concentric celestial spheres, rising and converging to a single luminous point (see Figure 11). Topologically, this finite yet unbounded space would today be described as a three-dimensional sphere.

In 1904, Poincare asked if the 3-sphere is the only closed 3-manifold in which every loop can be shrunk to a point; a positive answer became known as the Poincaré conjecture. Although the theory of manifolds developed rapidly in the following generations, this conjecture remained open.

In the 1980s, Thurston showed that a large class of 3-manifolds are hyperbolicthey admit rigid metrics of constant negative curvature. At the same time he proposed a geometric description of all 3-dimensional manifolds, subsuming the Poincaré conjecture as a special case.

Both the Poincaré conjecture and Thurston's geometrization conjecture have now been established through the work of Perelman. The confirmation of this achievement was recognized by a conference at the Institut Henri Poincaré in 2010.

Received by the editors October 17, 2010.

2010 Mathematics Subject Classification. Primary 57M50.

This research was supported in part by the NSF. 


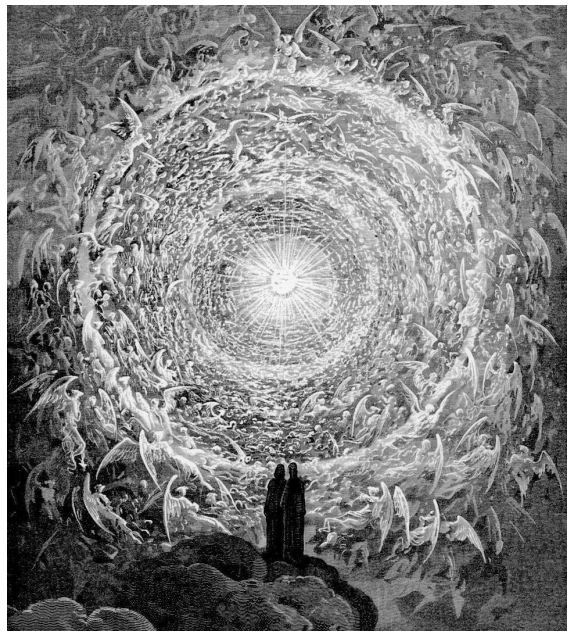

Figure 1. Illustration of Dante's cosmology by Gustave Doré (1867).

This article, based on a lecture at that conference, aims to give a brief and impressionistic introduction to the geometrization conjecture: its historical precedents, the approaches to its resolution, and some of the remaining open questions. Additional notes, and references to some of the many works treating these topics in detail, are collected at the end.

\section{Surfaces AND TILINGS}

We begin by recalling the geometrization theorem in dimension two.

Theorem 2.1. Any closed, orientable topological surface $S$ can be presented as a quotient of $S^{2}, \mathbb{E}^{2}$, or $\mathbb{H}^{2}$ by a discrete group of isometries.

Concretely, this means $S$ can be tiled by spherical, Euclidean, or hyperbolic polygons.

Proof. By the classification of surfaces, we may assume $S$ is a sphere, a torus, or a surface of genus $g \geq 2$. The theorem is immediate in the first two cases. In terms of tilings, one can assemble $S^{2}$ out of eight spherical triangles with all angles $90^{\circ}$; and a torus can be tiled by eight Euclidean squares, which unfold to give the checkerboard tiling of $\mathbb{E}^{2}$.

Next we observe that a surface of genus $g=2$ can be assembled from eight regular pentagons (see Figure 2). The right-angled pentagons needed for this tiling do not exist in spherical or Euclidean geometry, but they do exist in the hyperbolic plane. Passing to the universal cover $\tilde{S}$, we obtain a periodic tiling of $\mathbb{H}^{2}$ and an isometric action of the deck group $\Gamma \cong \pi_{1}(S)$ on $\mathbb{H}^{2}$ yielding $S$ as its quotient. Any surface of genus $g \geq 3$ covers a surface of genus 2 , so it too can be tiled by pentagons - one just needs more of them.

Uniformization. The geometrization theorem for surfaces was essentially known to Klein and his contemporaries in the 1870s, although the classification of abstract surfaces according to genus, by Dehn and Heegaard, was not proved until 1907. It is definitely more elementary than the uniformization theorem, proved in the same 


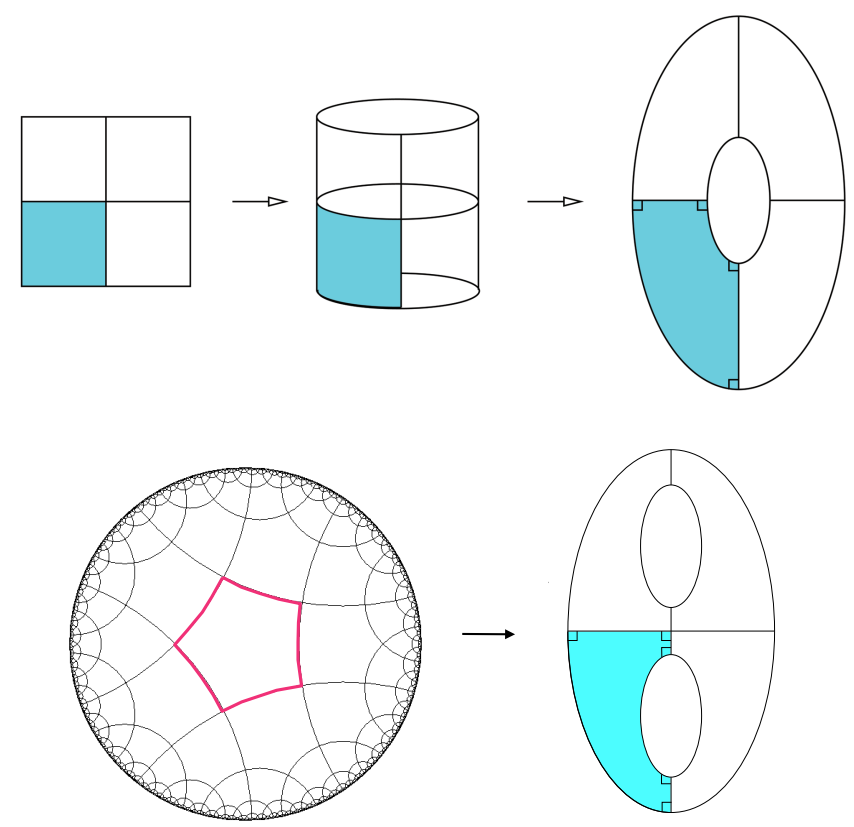

FIGURE 2. Tilings of surfaces of genus 1 and 2.

era, which asserts that every algebraic curve can be analytically parameterized by $\widehat{\mathbb{C}}, \mathbb{C}$, or $\mathbb{H}^{2}$.

The converse is also true, as was shown by Poincaré; in particular:

Theorem 2.2. Every compact Riemann surface of the form $X=\mathbb{H}^{2} / \Gamma$ is isomorphic to an algebraic curve.

For the proof, we need to construct meromorphic functions on $X$. A natural approach is to start with any rational function $f(z)$ on the unit disk $\Delta \cong \mathbb{H}^{2}$, and then make it invariant by forming the Poincaré series

$$
\Theta(f)=\sum_{\gamma \in \Gamma} \gamma^{*}(f)=\sum_{\gamma \in \Gamma} f(\gamma(z))
$$

The result can then be regarded as a meromorphic function on $X$.

Unfortunately this series has no chance of converging: the orbit $\gamma(z)$ accumulates on points in $\partial \Delta$ where $f(z) \neq 0$, so the terms in the sum do not even tend to zero.

However, the sum does converge if we replace the function $f(z)$ with the quadratic differential $q=f(z) d z^{2}$, since then $|q|$ behaves like an area form and the total area near the boundary of the disk is finite. This makes $\Theta(q)$ into a meromorphic form on $X$; and ratios of these forms, $\Theta\left(q_{1}\right) / \Theta\left(q_{2}\right)$, then give enough meromorphic functions to map $X$ to an algebraic curve.

Thus algebra, geometry, and topology are mutually compatible in dimension two. Poincaré's $\Theta$-operator also plays an unexpected role in the theory of 3 -manifolds; see $₫ 5$ 


\section{The GEOMETRIZATION CONJECTURE FOR 3-MANIFOLDS}

We now turn to the 3-dimensional case.

In contrast to the case of surfaces, which are ordered by genus, the world of 3-manifolds resembles an evolutionary tree, with phyla and species whose intricate variations admit, at best, a partial ordering by various measures of complexity.

An organizing principle for 3-manifolds seemed elusive until, in the 1980s, Thurston proposed the following:

Conjecture 3.1 (The geometrization conjecture). All compact 3-manifolds can be built using just eight types of geometry.

The eight geometries featured in this conjecture come from the following simply connected homogeneous spaces:

(1) The spaces of constant curvature, $S^{3}, \mathbb{E}^{3}$, and $\mathbb{H}^{3}$;

(2) The product spaces $\mathbb{R} \times S^{2}$ and $\mathbb{R} \times \mathbb{H}^{2}$; and

(3) The 3-dimensional Lie groups Nil, Sol, and $\mathrm{SL}_{2}(\mathbb{R})$.

A 3-manifold $M$ is geometric if it can be presented as the quotient $M=H / \Gamma$ of one of these homogeneous spaces by a discrete group of isometries. By gluing together geometric 3-manifolds along suitable spheres or tori, one obtains composite manifolds.

The geometrization conjecture states that any compact 3-manifold is either geometric or composite. In other words, any 3-manifold can be factored into "geometric primes".

Hyperbolic manifolds. Most of the eight geometries are only required to describe fairly simple 3-manifolds: products or twisted products of circles and surfaces (spaces of "dimension $2 \frac{1}{2}$ "). The manifolds covered by $S^{3}$ and $\mathbb{E}^{3}$ are also special - they are just finite quotients of the sphere or the 3-torus.

The only remaining case is that of hyperbolic manifolds - those of the form $M=$ $\mathbb{H}^{3} / \Gamma$. Thus a principal corollary of the geometrization conjecture is that most 3-manifolds are hyperbolic 1

On the other hand, since $S^{3}$ is the only closed, simply connected geometric 3-manifold, the geometrization conjecture also implies the Poincaré conjecture.

Spherical and hyperbolic dodecahedra. As in the case of dimension two, geometric 3-manifolds correspond to periodic tilings. Two such are shown in Figure 3 .

The first tiling comes from the Poincaré homology sphere $M=S^{3} / \Gamma$, where $\Gamma \cong \tilde{A}_{5}$ is a $\mathbb{Z} / 2$ extension of the alternating group on five symbols. A fundamental domain for the action of $\Gamma$ on $S^{3}$ is given by a spherical dodecahedron $D$. The faces of $D$ are regular pentagons meeting in angles of $120^{\circ}$; this allows three copies of $D$ to fit together neatly along an edge. (In Euclidean space, the angle would be about $116^{\circ}$.)

Poincaré originally speculated that the condition $H_{1}(M, \mathbb{Z})=0$ would be sufficient to characterize the 3 -sphere. The space $M=S^{3} / \Gamma$ just constructed (also discovered by Poincaré) provides a counterexample, since $\pi_{1}(M) \cong \tilde{A}_{5}$ abelianizes to the trivial group.

\footnotetext{
${ }^{1}$ A spatial universe of constant negative curvature (as would be consistent with a uniform distribution of matter and energy) can therefore have almost any global topological form.
} 

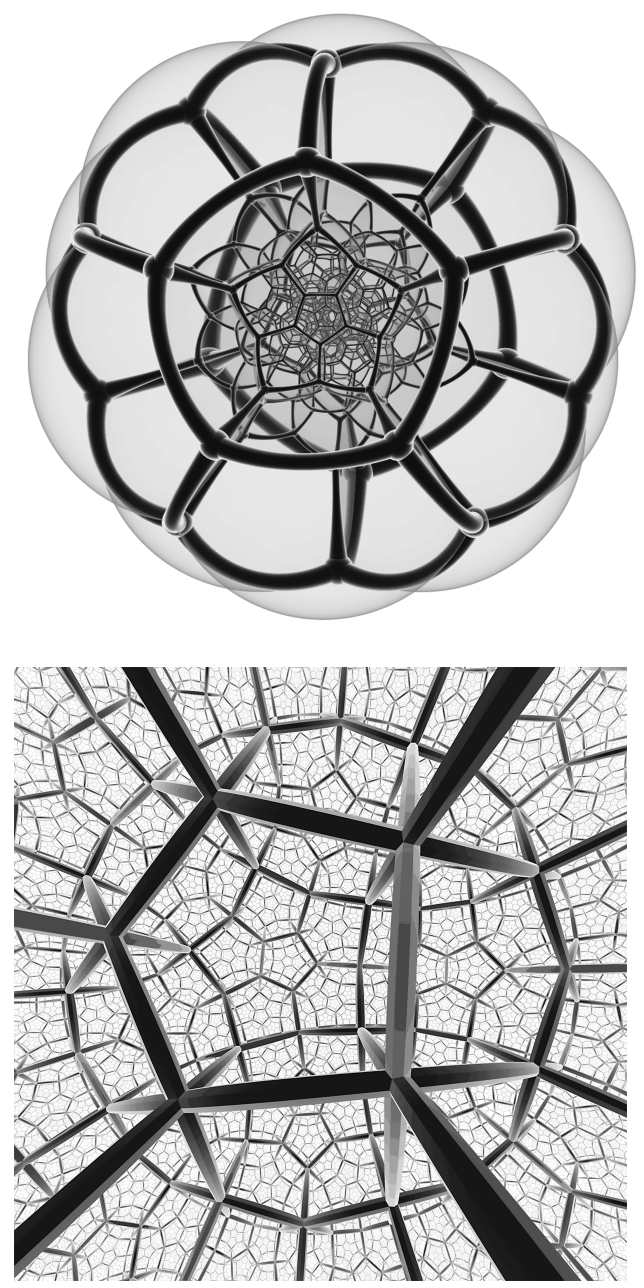

Figure 3. Tilings of $S^{3}$ and $\mathbb{H}^{3}$.

The second image in Figure 3 shows a tiling of $\mathbb{H}^{3}$ by infinitely many negatively curved dodecahedra. Now the dodecahedra meet four to an edge; their faces are the same right-angled pentagons that appeared in Figure 2. This pattern provides a hyperbolic metric on a closed 3-manifold $M$ that can be obtained as a 4 -fold cover of $S^{3}$ branched over the Borromean rings.

Arithmetic groups. Additional examples of hyperbolic 3-manifolds are provided by arithmetic subgroups of $\mathrm{SL}_{2}(\mathbb{C})$. The simplest of these are the Bianchi groups $\mathrm{SL}_{2}(\mathcal{O})$, where $\mathcal{O}=\mathbb{Z}[\sqrt{-d}]$ or $\mathbb{Z}[(1+\sqrt{-d}) / 2]$ is the ring of integers in a complex quadratic field.

The action of a Bianchi group by Möbius transformations on $\widehat{\mathbb{C}}$ extends to an isometric action on $\mathbb{H}^{3}$. Passing to a subgroup of finite index, we can ensure that $\Gamma \subset \mathrm{SL}_{2}(\mathcal{O})$ is torsion-free, and hence $M=\mathbb{H}^{3} / \Gamma$ is a manifold. General principles ensure that $M$ has finite volume, but it is never closed; instead, it is homeomorphic to the complement of a knot or link in some closed 3-manifold $\bar{M}$. 

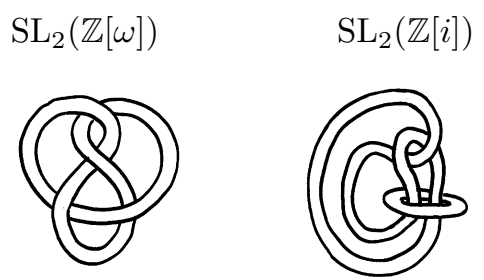

Figure 4. Arithmetic knots and links.

An important example is provided by the Eisenstein integers, $\mathcal{O}=\mathbb{Z}[\omega]$ where $\omega^{3}=1$. In this case $\Gamma \subset \mathrm{SL}_{2}(\mathbb{Z}[\omega])$ can be chosen so that $M=\mathbb{H}^{3} / \Gamma \cong S^{3}-K$ is the complement of the figure-eight knot in the sphere. Similarly, using $\mathcal{O}=\mathbb{Z}[i]$, one obtains an arithmetic hyperbolic structure on the complement of the Whitehead link (Figure 4).

These examples are related to tilings of $\mathbb{H}^{3}$ by regular ideal tetrahedra and octahedra, respectively.

Rigidity. The architectural integrity of the frameworks shown in Figure 3 and the arithmeticity of the preceding examples reflect an important feature of the passage from 2 to 3 dimensions: while topology becomes more flexible in higher dimensions, geometry becomes more rigid. A precise statement is furnished by:

Theorem 3.2 (Mostow Rigidity). The geometry of a finite-volume hyperbolic 3manifold is uniquely determined by its fundamental group.

Because of this uniqueness, geometric quantities such as the hyperbolic volume of $M^{3}$ or the length of its shortest geodesic are actually topological invariants. For example, the figure-eight knot satisfies

$$
\operatorname{vol}\left(S^{3}-K\right)=6 л(\pi / 3)=6 \int_{0}^{\pi / 3} \log \frac{1}{2 \sin \theta} d \theta=2.0298832 \cdots .
$$

The geometrization conjecture becomes even more striking when seen in light of this rigidity.

A comparison to number theory. The influence of the Poincaré conjecture on low-dimensional topology can be compared to the influence of Fermat's last theorem on number theory. Both conjectures have been driving forces in mathematics, but both their formulations are essentially negative.

The geometrization conjecture placed the Poincaré conjecture in the context of a comprehensive picture of 3-dimensional topology that could be tested and developed in many new directions. Similarly, work of Frey, Ribet, and Serre in the 1980s showed that Fermat's last theorem would follow from the modularity conjecture, which states:

Every elliptic curve $E$ defined over $\mathbb{Q}$ is dominated by a modular curve of the form $X_{0}(N)=\mathbb{H}^{2} / \Gamma_{0}(N)$.

Like the geometrization conjecture, the modularity conjecture is constructive and testable. For example, in 1993 Cremona calculated all the modular elliptic curves with conductor $N \leq 999$, lending support to the conjecture and furnishing important arithmetic invariants of these elliptic curves.

Some of the experimental work carried out for 3-manifolds will be discussed in the sections that follow. 


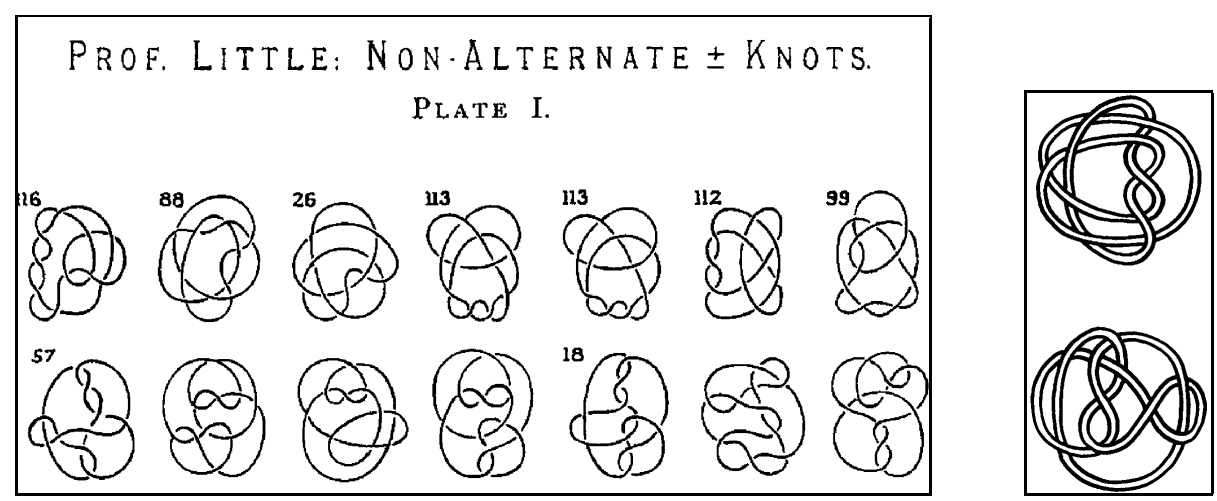

Figure 5. Tait and Little's knot tables (excerpt), 1899; the Perko pair, 1974.

\section{KNOTS}

What has become of all the simpler vortex atoms?

- P. G. Tait, 1876.

Knots and links provide a glimpse of the full complexity of 3-dimensional topology. In this section we discuss Thurston's results on hyperbolic 3-manifolds and their impact on knot theory.

Hyperbolic knots. A knot is a smoothly embedded circle in $S^{3}$. The union of finitely many disjoint knots is a link. By removing a thickened link in $S^{3}$ (a union of solid tori) and gluing it back in with a twist, one obtains a new 3-manifold $M$. Lickorish showed that all orientable 3 -manifolds can be obtained by surgery on links in $S^{3}[\mathrm{Li}$.

In the early 1980s Thurston established several major cases of the geometrization conjecture, including the following unexpected results:

(1) Almost all knots are hyperbolic;

(2) Almost all surgeries of $S^{3}$ along hyperbolic knots and links yield hyperbolic manifolds; and

(3) The result $M$ of gluing together two hyperbolic 3-manifolds is hyperbolic, unless $\pi_{1}(M)$ contains a copy of $\mathbb{Z}^{2}$.

Here a knot or link $L$ is hyperbolic if $S^{3}-L$ is homeomorphic to a finite volume hyperbolic manifold $\mathbb{H}^{3} / \Gamma$. In the first statement, just torus knots and satellite knots must be avoided; in the second, finitely many surgeries must be excluded on each component of the link. The third statement is the key to proving the first two; it will be taken up in 95 . All three results make precise, in various ways, the statement that most 3-manifolds are hyperbolic.

Tabulating knots. To put these results in context, we recount some history.

A knot can be conveniently described by a crossing diagram, showing its projection to a plane. Motivated by Lord Kelvin's theory of atoms as vortex rings, whose different shapes would account for the different chemical elements, in the period 1876-99 Tait and Little (aided by Kirkman) assembled a census of all 249 (prime) knots with 10 or fewer crossings (see e.g., Figure 5). It is a demanding but straightforward task to enumerate all such knot diagrams; the challenge is to tell when two different diagrams actually represent the same knot. 
In the 1960s J. H. Conway invented a more efficient combinatorial notation for knots, based on tangles. This notation allowed him to replicate the work by Tait and Little in a matter of days and to extend the existing tables to include all 552 knots with 11 crossings.

Both tables, however, contained a duplication: in 1974, the lawyer K. Perko discovered that the two diagrams shown at the right in Figure 5 actually represent the same knot.

Algorithms and geometry. With such pitfalls in mind, the prospect of proceeding further seemed daunting. Nevertheless, in 1998, Hoste, Thistlethwaite, and Weeks succeeded in tabulating all knots up to 16 crossings - all 1,701,936 of them HTW.

How was such a tabulation possible? Its cornerstone was a computer program, developed by Weeks, to find the hyperbolic structure on $M=S^{3}-K$. Although based on a heuristic algorithm, in practice this program almost always succeeds. The hyperbolic structure, in turn, yields a host of numerical invariants for $K$, such as the volume of $M=S^{3}-K$; and it also provides a canonical triangulation of $M$ (dual to a fundamental domain "centered" on $K$ ). This triangulation is a complete invariant of $K$, so it suffices to eliminate all duplicate hyperbolic knots. (In particular, the algorithm immediately recognizes the Perko pair as two diagrams for the same knot.)

We remark that the practical computation of hyperbolic structures for knots, while motivated by Thurston's results, does not logically rely upon them; nor do the existing proofs of the existence of hyperbolic structures yet explain why such computations are so robust.

\section{EvOlving GEOMETRIC STRUCTURES}

We now turn to the proof of the geometrization conjecture. We will discuss two important processes for deforming a topological 3-manifold towards its optimal geometric shape: conformal iteration and the Ricci flow.

1. Haken manifolds. We begin with some terminology. Let $M$ be a compact orientable 3-manifold, possibly with boundary. A connected orientable surface $S \subset$ $M^{3}$ is incompressible if $S \neq S^{2}$ and $\pi_{1}(S)$ maps injectively into $\pi_{1}(M)$.

A 3-manifold is Haken if it can be built up, starting from 3-balls, by successively gluing along incompressible submanifolds of the boundary. Any knot or link complement is Haken, as is any irreducible 3-manifold with boundary. Thus most of the results stated in $\$ 4$ for knots are consequences of:

Theorem 5.1 (Thurston). The geometrization conjecture holds for Haken 3-manifolds.

Iteration on Teichmüller space. Since the seven simpler geometries are understood for Haken manifolds, the main point in the proof of Theorem 5.1 is to treat the hyperbolic case. At the critical inductive step, one has an open hyperbolic 3manifold $M$ with incompressible boundary, and a gluing involution $\tau: \partial M \rightarrow \partial M$ 


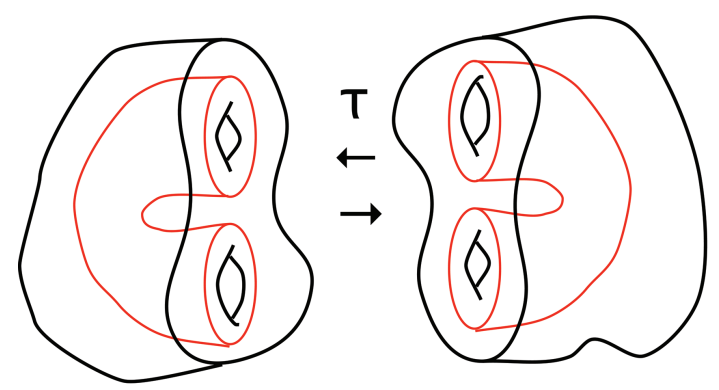

FiguRE 6. Gluing succeeds unless it is obstructed by a torus.

(see Figure 6). The task is to produce a hyperbolic metric on the closed manifold $M / \tau$.

A generalization of Mostow rigidity shows that hyperbolic structures on the interior of $M$ correspond to conformal structures on $\partial M$. They are therefore parameterized by Teichmüller space, a finite-dimensional complex manifold homeomorphic to a ball. Thurston showed a solution to the gluing problem corresponds to a fixed point for a topologically defined holomorphic map

$$
\sigma \circ \tau: \operatorname{Teich}(\partial M) \rightarrow \operatorname{Teich}(\partial M)
$$

By iterating this map, we obtain an evolving sequence of hyperbolic structures on $M$. If the sequence converges, then $M / \tau$ is hyperbolic.

One obstruction to convergence comes from $\pi_{1}(M / \tau)$ : the fundamental group of a closed, negatively curved manifold never contains a copy of $\mathbb{Z}^{2}$. In fact, as Thurston showed, this is the only obstruction.

Theorem 5.2. $M / \tau$ is hyperbolic $\Longleftrightarrow \pi_{1}(M / \tau)$ does not contain $\mathbb{Z}^{2}$.

Sketch of the proof. We describe an approach based on complex analysis developed in Mc1. At a given point $X \in \operatorname{Teich}(\partial M)$, the Poincaré series operator introduced in $\$ 2$ provides a map $\Theta_{X}: Q(\Delta) \rightarrow Q(X)$ from $L^{1}$ holomorphic quadratic differentials on the disk to those on $X$. It turns out the norm of the operator depends only on the location of $X$ in moduli space and satisfies $\left\|\Theta_{X}\right\|<1$. Using the fact that $Q(X)$ forms the cotangent space to Teichmüller space at $X$, one can also show that $\sigma \circ \tau$ is a contraction in the Teichmüller metric with the bound

$$
\left|(\sigma \circ \tau)^{\prime}(X)\right| \leq\left\|\Theta_{X}\right\|<1 .
$$

Now start with an arbitrary Riemann surface $X_{0} \in \operatorname{Teich}(\partial M)$ and form the sequence

$$
X_{n}=(\sigma \circ \tau)^{n}\left(X_{0}\right) .
$$

Then the bound above shows we have uniform contraction - and hence convergence to a fixed point-unless $\left[X_{n}\right]$ tends to infinity in moduli space. But in this case $X_{n} \cong \partial M$ develops short geodesics, which bound cylinders in $M$ that are joined together by $\tau$ to yield a torus in $M / \tau$ as shown in Figure 6. Thus $\pi_{1}(M / \tau)$ contains the obstruction $\mathbb{Z}^{2}$.

This gluing construction is the pivotal step in Thurston's proof of Theorem 5.1. It also resonates with similar approaches to the topology of rational maps, the classification of surface diffeomorphisms and Mordell's conjecture in the function field case; see e.g., [DH], [Mc2], Mc5]. 
For simplicity we have assumed $M$ is not a virtual product, $S \times[0,1]$. The gluing theorem remains true in this case, but a somewhat different proof is required.

2. Evolution by curvature. We now turn to the second approach, used by Perelman to complete the proof of the geometrization conjecture.

Darwin recognized that his weak and negative force... could only play $[a]$ creative role if variation met three crucial requirements: copious in extent, small in range of departure from the mean, and isotropic.

- S. J. Gould.

In 1982, Hamilton introduced the Ricci flow

$$
\frac{d g_{i j}}{d t}=-2 R_{i j}
$$

for an evolving Riemannian metric $g_{i j}$ on a manifold $M$. This is a (nonlinear) heat-equation type flow driven by the Ricci tensor, a contraction of the Riemann curvature form that also plays a central role in general relativity.

The idea of the Ricci flow is shown in Figure 7 , here, an initial space of variable curvature evolves continuously until it becomes recognizable as a round circle. The manifold bends in response to its own shape, continuously adapting so that as $t \rightarrow \infty$ a metric of constant curvature may emerge.

Hamilton made several pioneering contributions to geometrization using the Ricci flow, including a proof of the Poincaré conjecture for manifolds with positive Ricci curvature [Ham1]. In this case the manifold shrinks to a point in finite time, but under rescaling it converges to a round unit sphere.

We remark that the Ricci flow, like natural selection itself, satisfies the three principles enunciated by Gould: as a differential equation on the whole manifold, it is copious in extent but small in departure from the mean; and it is isotropic, since the Ricci curvature is an intrinsic invariant of the metric.
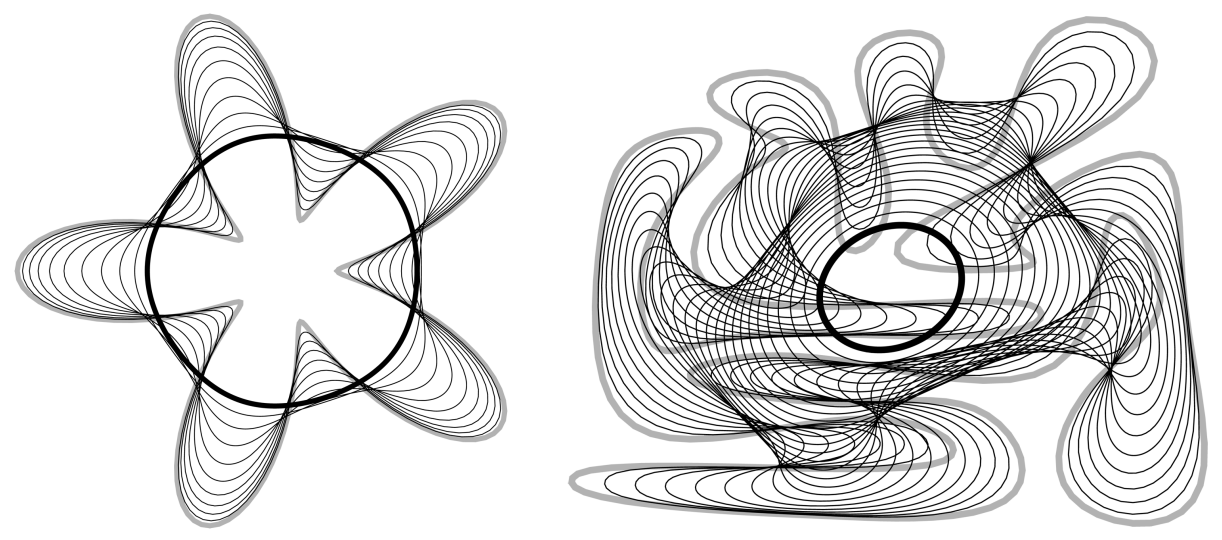

Figure 7. Curvature flow.

Perelman's work. There are two main obstacles to long-term evolution under the Ricci flow: singularities may develop, which rapidly pinch off and break the manifold into pieces, and the manifold may collapse - it may become filled with short loops, even though its curvature remains bounded. 
Perelman's work addresses both of these obstacles, and indeed turns them into the cornerstones of a successful proof of the geometrization conjecture. In brief, he shows that in dimension 3:

(1) Singularities of the Ricci flow always occur along shrinking 2-spheres, which split $M$ into a connected sum of smaller pieces. These singularities can be sidestepped by an explicit surgery operation.

(2) Curvature evolution with surgery defines a flow which continues for all time.

(3) In the limit as $t \rightarrow \infty$, a geometric structure on the pieces of $M$ becomes visible, either through convergence to a metric of constant curvature or through collapsing.

As a consequence we have:

Theorem 5.3 (Perelman). Both the Poincaré conjecture and the geometrization conjecture are true.

Comparison. Many detailed accounts of Perelman's work are now available in the literature. Here we will only add a few comparisons between these two different evolutionary processes.

(1) The discrete dynamics of conformal iteration takes place on the finitedimensional space of hyperbolic manifolds. It proceeds through a sequence of classical, finite-sided hyperbolic polyhedra with varying shapes, converging to a form suitable for gluing.

The continuous Ricci flow, on the other hand, takes place in the infinitedimensional space of smooth metrics. Constant curvature and homogeneous geometry emerge only in the limit.

(2) Iteration on Teichmüller space is a contraction, and hence guaranteed to converge if a fixed point exists. In this way it leverages Mostow rigidity (which implies the fixed point is unique).

The analysis of the Ricci flow, on the other hand, pivots on monotonicity. Various entropy-like quantities increase under the flow, allowing one to obtain compactness results and to rule out breathers (oscillating solutions to the flow which cannot possibly converge).

Rigidity or uniqueness of the limiting geometry is not apparent from this perspective.

(3) The approach for Haken manifolds is bottom-up: the geometry of $M$ is assembled inductively from smaller geometric pieces by cutting along a hierarchy of surfaces.

The Ricci flow approach is top-down; the metric evolves on the manifold as a whole, splitting it into pieces as singularities develop. Thus it can be applied to 3-manifolds which are too tightly wound (or too homotopically simple) to contain an incompressible surface.

Because of these features, the evolutionary approach based on the Ricci flow is able to treat the geometrization conjecture in full.

Remark (The cone-manifold approach). By Thurston's Theorem[5.1, any 3-manifold contains a knot such that $M^{3}-K$ is hyperbolic. One can then try to increase the cone angle along the knot from 0 to $360^{\circ}$ to obtain a geometric structure on $M$. This cone-manifold approach to geometrization works well for constructing orbifolds (see e.g., $[\mathrm{CHK}]$ ), but it runs into difficulties, still unresolved, when the strands of the knot collide. 
The Ricci flow, on the other hand, smooths out such conical singularities, diffusing the knot so it can freely pass through itself.

\section{Open PROBLEMS}

To conclude, we mention two of the many remaining open problems in the theory of 3-manifolds.

1. Surfaces in 3-manifolds. As we have seen, a useful approach to simplifying a 3-manifold involves cutting it open along an incompressible surface. A central problem, still open, is to understand how often such surfaces exist; in particular, to establish:

Conjecture 6.1 (Waldhausen, 1968). Every closed, irreducible 3-manifold $M$ with infinite fundamental group has a finite cover which contains an incompressible surface.

Part of the impact of the proof of the geometrization conjecture is that it allows topological problems to be studied by geometric means. For example, in the conjecture above, one may now assume $M$ is hyperbolic.

In 2003, Dunfield and Thurston verified Conjecture 6.1 for the more than 10,000 different hyperbolic 3-manifolds appearing in the Hodgson-Weeks census [DT]. Further progress includes the following result from 2010:

Theorem 6.2 (Kahn-Markovic). If $M$ is a closed hyperbolic 3-manifold, then $\pi_{1}(M)$ contains a surface group.

The proof uses ergodic theory on the frame bundle of $M$ to analyze statistical properties of the pairs of pants it contains, which are then pieced together (in enormous numbers) to form a closed, immersed surface [KM].

It remains a challenge to find a finite cover where this surface becomes embedded.

2. Quantum topology. The curved space of general relativity becomes a sea of virtual particles when viewed through the lens of quantum mechanics. Similarly, quantum topology gives a new perspective on 3-manifolds.

An example is provided by the knot polynomial $V(K, t)$ discovered by Jones in 1984. The Jones polynomial can be computed from a knot diagram by a simple inductive procedure, but it proves difficult to say what $V(K, t)$ measures in terms of classical topology.

On the other hand, Witten found a useful description in terms of physics: in 1988 he proposed that for each integer $k \geq 0$, the value of $V(K, t)$ at the root of unity $q=\exp (2 \pi i /(2+k))$ should satisfy the relation:

$$
\langle K\rangle=\int \operatorname{Tr}\left(\int_{K} A\right) e^{2 \pi i k \operatorname{CS}(A)} D A=\left(q^{1 / 2}+q^{-1 / 2}\right) V\left(K, q^{-1}\right) .
$$

Here $A$ is an $\mathrm{SU}(2)$-connection on the trivial $\mathbb{C}^{2}$ bundle over $S^{3}$. The factor $e^{2 \pi i k \operatorname{CS}(A)} D A$ represents a formal probability measure on the space of all connections, coming from quantum field theory and the Chern-Simons action. Finally $\langle K\rangle$ is the expected value of the random variable $\operatorname{Tr}\left(\int_{K} A\right)$, which measures the twisting of the connection along the knot.

Many additional invariants of low-dimensional manifolds have emerged from the perspective of quantum field theory in recent decades and have been made rigorous using combinatorial methods and gauge theory. 
How might these developments relate to the geometrization conjecture? A possible connection is provided by:

Conjecture 6.3 (Kashaev, Murakami-Murakami). The hyperbolic volume of $S^{3}-$ $K$ can be calculated using the Jones polynomials of the cables of $K$; in fact, we have

$$
\operatorname{vol}\left(S^{3}-K\right)=\lim _{n \rightarrow \infty} \frac{2 \pi \log \left|V_{n}\left(K, e^{2 \pi i / n}\right)\right|}{n} .
$$

Here $V_{n}(K, t)=\sum_{j=0}^{n / 2}\left(\begin{array}{c}n-j \\ j\end{array}\right) V\left(K^{n-2 j}, t\right)$, where $K^{i}$ is the cabled link formed by $i$ parallel copies of $K$.

The idea behind this conjecture is that the $\mathrm{SU}(2)$ connections on $S^{3}-K$ should be sensitive to the flat $\mathrm{SL}_{2}(\mathbb{C})$ connection defining its hyperbolic structure.

At present, the volume conjecture above has been verified for only a handful of knots, including the figure-eight knot. It hints, however, at a deeper connection between geometric and quantum topology, mediated perhaps by the multitude of fluctuating combinatorial descriptions that a single geometric manifold can admit.

\section{Notes AND REFERENCES}

$\S 1$. For a historical perspective on the Poincaré conjecture, see Mil.

$\S 2$, Poincaré's works on Fuchsian groups and $\Theta$-series are collected in $\mathrm{Po}$.

$\S 3$. The geometrization conjecture is formulated in [Th1. For more on the eight 3-dimensional geometries, see e.g., $\mathrm{Sc}$ and [Th5].

A variant of the hyperbolic tiling shown in Figure 3, in which five dodecahedra meet along an edge, was discovered by Seifert and Weber in 1933 [SW]. Seifert and Weber also related their example to the Poincaré sphere and to a covering of $S^{3}$ branched over the Whitehead link. The graphics in Figure 3 were produced by Fritz Obermeyer and by the Geometry Center.

It is known that the figure-eight knot is the only arithmetic knot $[\mathbf{R e}$. Additional arithmetic links are described in Hat.

The original proof of Mostow rigidity (generalized to manifolds of finite volume by Prasad) was based on ergodic theory and quasiconformal mappings [Mos. For a more geometric proof, due to Gromov, see e.g., [Rat, §11].

A discussion of the modularity conjecture and Fermat's last theorem can be found in Mazur's article Maz. The proof of the modularity conjecture was completed in 2001, through work of Breuil, Conrad, Diamond, Taylor, and Wiles.

$\S 4$. Thurston's results are presented in [Th1]. In the 1990s, Casson and Jungreis CJ] and Gabai Ga made important progress on the seven nonhyperbolic geometries by characterizing Seifert fiber spaces.

The work of Tait appears in [Ta and its sequels. Figure 5 is taken from Little's paper Lit]. These authors had no rigorous methods even to distinguish the trefoil knot from the unknot. The success of their tabulations, especially for alternating knots, is due in part to the validity of the Tait conjectures, which were finally proved using the Jones polynomial (see e.g., MT]). One of these conjectures asserts that the writhe of a reduced alternating diagram is an invariant of the knot; the Perko pair shows this is false for nonalternating knots.

Conway's work on knots up to 11 crossings appears in Con.

$\S 5$. Thurston's proof of the geometrization conjecture for Haken manifolds is outlined in [Mor1]. Large portions appear in [Th2, Th3, and Th4]. An orbifold 
construction allows one to reduce to the case where gluing is along the full boundary of $M$; some additional work is required to keep track of the parabolic locus. A complete proof is presented by Kapovich in Kap. The case of 3 -manifolds that fiber over the circle, which requires a different gluing argument, is treated in detail in [Th3 and $\mathrm{Ot}$; see also [Mc4. The analytic proof of the gluing step presented here appears in Mc1]; see also Mc3.

Gould's statement on natural selection is taken from Go, p.60]. Figure 7 actually depicts two examples of the mean curvature flow for hypersurfaces, a variant of the Ricci flow studied in dimension two by Gage and Hamilton [GH]; see also [Gr]. For Hamilton's work on the Ricci flow for 3-manifolds, see Ham1, Ham2, and Ham3.

Perelman's proof, which appeared in Per1, Per2, and Per3, is surveyed in [And, [Mor2], and [Be], and presented in detail in [KL], [MT1], and [CZ]; see also the forthcoming books [MT2] and [BMP].

$\S$ 6. For more on the Jones polynomial and quantum topology, see e.g., [J1, [J2, Wit] and Atiyah's book [At]. The volume conjecture is formulated in [Ka] and MM] see also the survey Mur.

\section{ABOUT THE AUTHOR}

Curtis T. McMullen is a professor of mathematics at Harvard University.

\section{REFERENCES}

[And] M. T. Anderson. Geometrization of 3-manifolds via the Ricci flow. Notices Amer. Math. Soc. 51 (2004), 184-193. MR2026939 (2004i:53095)

[At] M. Atiyah. The Geometry and Physics of Knots. Cambridge University Press, 1990. MR.1078014 (92b:57008)

[BMP] L. Bessières, G. Besson, Michel Boileau, S. Maillot, and J. Porti. Geometrisation of 3Manifolds. In preparation.

[Be] G. Besson. Preuve de la conjecture de Poincaré en déformant la métrique par la courbure de Ricci (d'après G. Perel'man). In Séminaire Bourbaki 2004/2005, pages 309-347. Astérisque, vol. 307, 2006. MR2296423 (2008b:53088)

[CZ] H.-D. Cao and X.-P. Zhu. A complete proof of the Poincaré and geometrization conjectures - application of the Hamilton-Perelman theory of the Ricci flow. Asian J. Math. 10 (2006), 165-492. MR2233789 (2008d:53090)

[CJ] A. Casson and D. Jungreis. Convergence groups and Seifert fibered 3-manifolds. Invent. Math. 118 (1994), 441-456. MR.1296353 (96f:57011)

[Con] J. H. Conway. An enumeration of knots and links, and some of their algebraic properties. In Computational Problems in Abstract Algebra (Proc. Conf., Oxford, 1967), pages 329358. Pergamon, 1970. MR0258014 (41:2661)

[CHK] D. Cooper, C. D. Hodgson, and S. P. Kerckhoff. Three-Dimensional Orbifolds and ConeManifolds. Mathematical Society of Japan, 2000. MR1778789(2002c:57027)

[DH] A. Douady and J. Hubbard. A proof of Thurston's topological characterization of rational maps. Acta Math. 171 (1993), 263-297. MR1251582 (94j:58143)

[DT] N. Dunfield and W. Thurston. The virtual Haken conjecture: experiments and examples. Geometry and Topology 7 (2003), 399-441. MR1988291 (2004i:57024)

[Ga] D. Gabai. Convergence groups are Fuchsian groups. Annals of Math. 136 (1992), 447-510. MR.1189862 (93m:20065)

[GH] M. Gage and R. S. Hamilton. The heat equation shrinking convex plane curves. J. Differential Geom. 23 (1986), 69-96. MR.840401 (87m:53003)

[Go] S. J. Gould. The Structure of Evolutionary Theory. Harvard University Press, 2002.

[Gr] M. A. Grayson. The heat equation shrinks embedded plane curves to round points. $J$. Differential Geom. 26 (1987), 85-314. MR906392(89b:53005) 
[Ham1] R. S. Hamilton. Three manifolds with positive Ricci curvature. J. Diff. Geom. 17 (1982), 255-306. MR664497 (84a:53050)

[Ham2] R. S. Hamilton. The formation of singularities in the Ricci flow. In Surveys in Differential Geometry, Vol. II (Cambridge, MA, 1993), pages 7-136. Int. Press, 1995. MR 1375255 (97e:53075)

[Ham3] R. S. Hamilton. Non-singular solutions of the Ricci flow on three-manifolds. Comm. Analysis and Geometry 7 (1999), 695-729. MR.1714939 (2000g:53034)

[Hat] A. Hatcher. Hyperbolic structures of arithmetic type on some link complements. J. London Math. Soc. 27 (1983), 345-355. MR692540 (84m:57005)

[HTW] J. Hoste, M. Thistlethwaite, and J. Weeks. The first 1,701,936 knots. Math. Intelligencer 20 (1998), 33-48. MR 1646740 (99i:57015)

[J1] V. F. R. Jones. Hecke algebra representations of braid groups and link polynomials. Ann. of Math. 126 (1987), 335-388. MR.908150 (89c:46092)

[J2] V. F. R. Jones. On the origin and development of subfactors and quantum topology. Bull. Amer. Math. Soc. 46 (2009), 309-326. MR2476415 (2009k:46113)

[KM] J. Kahn and V. Markovic. Immersing almost geodesic surfaces in a closed hyperbolic 3-manifold. Preprint, 2010.

[Kap] M. Kapovich. Hyperbolic Manifolds and Discrete Groups. Birkhäuser Boston, 2001. MR:1792613 (2002m:57018)

[Ka] R. M. Kashaev. The hyperbolic volume of knots from the quantum dilogarithm. Lett. Math. Phys. 39 (1997), 269-275. MR1434238(98b:57012)

[KL] B. Kleiner and J. Lott. Notes on Perelman's papers. Geom. Topol. 12 (2008), 2587-2855. MR 2460872(2010h:53098)

[Li] W. B. R. Lickorish. Representation of orientable combinatorial 3-manifolds. Annals of Mathematics 76 (1962), 531-540. MR0151948 (27:1929)

[Lit] C. N. Little. Non-alternate +/- knots. Trans. Royal. Soc. Edinburgh 39 (1898-9), 771778.

[Maz] B. Mazur. Number theory as gadfly. Amer. Math. Monthly 98 (1991), 593-610. MR.1121312 (92f:11077)

[Mc1] C. McMullen. Iteration on Teichmüller space. Invent. Math. 99 (1990), 425-454. MR.1031909 (91a:57008)

[Mc2] C. McMullen. Rational maps and Kleinian groups. In Proceedings of the International Congress of Mathematicians (Kyoto, 1990), pages 889-900. Springer-Verlag, 1991. MR:1159274 (93h:57024)

[Mc3] C. McMullen. Riemann surfaces and the geometrization of 3-manifolds. Bull. Amer. Math. Soc. 27 (1992), 207-216. MR1153266 (93b:57013)

[Mc4] C. McMullen. Renormalization and 3-Manifolds which Fiber over the Circle, volume 142 of Annals of Math. Studies. Princeton University Press, 1996. MR1401347 (97f:57022)

[Mc5] C. McMullen. From dynamics on surfaces to rational points on curves. Bull. Amer. Math. Soc. 37 (2000), 119-140. MR.1713286 (2000j:11093)

[MT] W. Menasco and M. Thistlethwaite. The Tait flyping conjecture. Bull. Amer. Math. Soc. 25 (1991), 403-412. MR1098346 (92b:57017)

[Mil] J. Milnor. Towards the Poincaré conjecture and the classification of 3-manifolds. Notices Amer. Math. Soc. 50 (2003), 1226-1233. MR2009455(2004h:57022)

[Mor1] J. Morgan. On Thurston's uniformization theorem for three dimensional manifolds. In The Smith Conjecture, pages 37-125. Academic Press, 1984. MR758464

[Mor2] J. Morgan. Recent progress on the Poincaré conjecture and the classification of 3manifolds. Bull. Amer. Math. Soc. 42 (2005), 57-78. MR2115067(2005i:57015)

[MT1] J. Morgan and G. Tian. Ricci Flow and the Poincaré Conjecture. Amer. Math. Soc., 2007. MR 2334563(2008d:57020)

[MT2] J. Morgan and G. Tian. Ricci Flow and the Geometrization Conjecture. In preparation.

[Mos] D. Mostow. Strong Rigidity of Locally Symmetric Spaces, volume 78 of Annals of Math. Studies. Princeton University Press, 1972.

[Mur] H. Murakami. A quantum introduction to knot theory. In Primes and Knots, volume 416 of Contemp. Math., pages 137-165. Amer. Math. Soc., 2006. MR2276140 (2008f:57018)

[MM] H. Murakami and J. Murakami. The colored Jones polynomials and the simplicial volume of a knot. Acta Math. 186 (2001), 85-104. MR.1828373 (2002b:57005) 
[Ot] J.-P. Otal. Le théorème d'hyperbolisation pour les variétés fibrées de dimension trois. Astérisque, vol. 235, 1996. MR1402300 (97e:57013)

[Per1] G. Perelman. The entropy formula for the Ricci flow and its geometric applications. arXiv:math/0211159.

[Per2] G. Perelman. Ricci flow with surgery on three-manifolds. arXiv:math/0303109.

[Per3] G. Perelman. Finite extinction time for the solutions to the Ricci flow on certain threemanifolds. arXiv:math/0307245.

[Po] H. Poincaré. Oeuvres. Tome II: Fonctions fuchsiennes. Éditions Jacques Gabay, 1995. MR:1401791 (97c:01053)

[Rat] J. G. Ratcliffe. Foundations of Hyperbolic Manifolds. Springer-Verlag, 1994. MR.1299730 (95j:57011)

[Re] A. W. Reid. Arithmeticity of knot complements. J. London Math. Soc. 43 (1991), 171184. MR.1099096 (92a:57011)

[Sc] P. Scott. The geometries of 3-manifolds. Bull. London Math. Soc. 15 (1983), 401-487. MR.705527 (84m:57009)

[SW] H. Seifert and C. Weber. Die beiden Dodekaederräume. Math. Z. 37 (1933), 237-253. MR.1545392

[Ta] P. G. Tait. On knots I. Trans. Roy. Soc. Edinburgh 28 (1876-7), 145-190.

[Th1] W. P. Thurston. Three-dimensional manifolds, Kleinian groups and hyperbolic geometry. Bull. Amer. Math. Soc. 6 (1982), 357-381. MR648524 (83h:57019)

[Th2] W. P. Thurston. Hyperbolic structures on 3-manifolds I: Deformations of acylindrical manifolds. Annals of Math. 124 (1986), 203-246. MR855294(88g:57014)

[Th3] W. P. Thurston. Hyperbolic structures on 3-manifolds II: Surface groups and 3-manifolds which fiber over the circle. Preprint, 1986. MR855294 (88g:57014)

[Th4] W. P. Thurston. Hyperbolic structures on 3-manifolds III: Deformation of 3-manifolds with incompressible boundary. Preprint, 1986. MR855294 (88g:57014)

[Th5] W. P. Thurston. Three-Dimensional Geometry and Topology, volume 1. Princeton University Press, 1997. MR:1435975 (97m:57016)

[Wit] E. Witten. Quantum field theory and the Jones polynomial. Commun. Math. Phys. 121 (1989), 351-399. MR990772 (90h:57009)

Department of Mathematics, Harvard University, 1 Oxford Street, Cambridge, MasSACHUSETTS 02138-2901 